\title{
Investigation Into Laser Shock Processing
}

\author{
B.S. Yilbas, A.F.M. Arif, S.Z. Shuja, M.A. Gondal, and J. Shirokof
}

(Submitted 26 March 2002; in revised form 17 September 2002)

\begin{abstract}
Laser shock processing is a good candidate for surface industry due to its rapid processing, localized ablation, and precision of operation. In the current study, laser shock processing of steel was considered. The numerical solutions for temperature rise and recoil pressure development across the interface of the ablating front and solid are presented. The propagation of elastic-plastic waves in the solid due to recoil pressure loading at the surface is analyzed and numerical solution for the wave propagation was obtained. An experiment was conducted to ablate the steel surfaces for shock processing. Scanning electron microscopy was carried out to examine the ablated surfaces shock processing while transmission electron microscopy was conducted to obtain dislocation densities after the shock processing. It was found that surface hardness of the workpiece increased in the order of 1.8 times of the base material hardness, and the dislocation was the main source of the shock hardening in the region affected by laser shock processing.
\end{abstract}

Keywords ablation, laser, processing, shock

\section{Introduction}

Laser shock processing of metallic surfaces is one of the interesting research fields in material processing technology, since laser shock processing is limited to the region that can be treated locally. Laser shock processing is involved with ablation of the surface. In this case, an incident laser beam is absorbed in the surface region of the substrate material. Once the amount of energy absorbed in this region exceeds the latent heat of evaporation of the substrate material, ablation initiates. Since the ablating front velocity is high, the recoil pressure is generated at the interface of the ablated front and the solid surface. Depending on the magnitude of recoil pressure, a stress wave propagating into the substrate material is developed. When the stress level exceeds the yielding limit of the substrate material, plastic deformation occurs. The formation of excessive arrays of dislocations and the formation of other phases or twins in the plastic region cause hardening of the substrate material. Moreover, once the ablation diminishes, the unloading elastic wave is generated, which propagates toward the solid bulk. The elastic wave propagates faster than the plastic wave and it meets with the plastic wave at some depth below the surface. The depth at which both waves met defines the plastic region of the substrate material. However, the use of Q-switched pulses ( 150 ps) may have disadvantages over the nanosecond laser pulses. This is due to that the rapid relaxation of the plastic wave by release-waves (elastic waves) immediately after the pulse terminates. This decreases the propagation depth of the shock wave inside the material; therefore, the desired effect is limited to a very shal-

B.S. Yilbas, A.F.M. Arif, and S.Z. Shuja, ME Department, KFUPM, Dhahran, Saudi Arabia; M.A. Gondal, Laser Research Section, Center For Applied Physical Sciences, KFUPM, Dhahran, Saudi Arabia; and J. Shirokof, Faculty of Engineering and Applied Science, Memorial University of Newfoundland, Canada. Contact e-mail: bsyilbas@kfupm.edu.sa. low depth. Consequently, nanosecond pulses have the advantage in producing a considerably larger depth of the shockaffected zone.

\begin{tabular}{|c|c|}
\hline & Nomenclature \\
\hline$A$ & area \\
\hline$c_{\mathrm{o}}$ & elastic wave speed, $\mathrm{m} / \mathrm{s}$ \\
\hline$c_{1}$ & plastic wave speed, $\mathrm{m} / \mathrm{s}$ \\
\hline$C_{\mathrm{p}}$ & specific heat, J/kg.K \\
\hline E & elastic modulus, $\mathrm{Pa}$ \\
\hline$h$ & plastic factor \\
\hline$H$ & hardness, HV \\
\hline$I_{\mathrm{o}}$ & laser peak power intensity, $\mathrm{W} / \mathrm{m}^{2}$ \\
\hline$k$ & thermal conductivity, W/m.K \\
\hline$k_{\mathrm{B}}$ & Boltzmann's constant, $1.38 \times 10^{-23} \mathrm{~J} / \mathrm{K}$ \\
\hline$L_{\mathrm{ev}}$ & latent heat of evaporation, $\mathrm{J} / \mathrm{kg}$ \\
\hline$m$ & atomic mass, $\mathrm{kg}$ \\
\hline$P$ & dislocation density, $1 / \mathrm{cm}^{2}$ \\
\hline$P_{\mathrm{r}}$ & Recoil pressure, $\mathrm{Pa}$ \\
\hline$\dot{q}_{\text {solid }}$ & energy dissipated in the solid phase, $\mathrm{W} / \mathrm{m}^{2}$ \\
\hline$r_{\mathrm{f}}$ & reflection coefficient \\
\hline$T(x, t)$ & temperature, $\mathrm{K}$ \\
\hline$T_{\mathrm{s}}$ & surface temperature, $\mathrm{K}$ \\
\hline$T_{\mathrm{ev}}$ & evaporation temperature, $\mathrm{K}$ \\
\hline$t$ & time, $\mathrm{s}$ \\
\hline$t_{\mathrm{p}}$ & Twice of pulse length, $18 \mathrm{~ns}$ \\
\hline$u$ & velocity in $x$-direction, $\mathrm{m} / \mathrm{s}$ \\
\hline$U$ & displacement, $\mathrm{m}$ \\
\hline$V$ & recession velocity of the surface, $\mathrm{m} / \mathrm{s}$ \\
\hline$V_{\mathrm{ev}}$ & vapor front velocity, $\mathrm{m} / \mathrm{s}$ \\
\hline$x$ & $\begin{array}{l}\text { spatial coordinates corresponding to the } x, y, z \text { axes } \\
\text { for phonon, } \mathrm{m}\end{array}$ \\
\hline$\alpha$ & thermal diffusivity, $\mathrm{m}^{2} / \mathrm{s}$ \\
\hline$\beta$ & laser pulse parameter, $1 / \mathrm{s}$ \\
\hline$\delta$ & absorption coefficient, $1 / \mathrm{m}$ \\
\hline$\varepsilon$ & strain \\
\hline$\kappa$ & yield stress, $\mathrm{Pa}$ \\
\hline$\kappa_{\mathrm{o}}$ & elastic yield limit, $\mathrm{Pa}$ \\
\hline$\lambda$ & mean free path of electrons, $\mathrm{m}$ \\
\hline$\rho$ & density, $\mathrm{kg} / \mathrm{m}^{2}$ \\
\hline
\end{tabular}


Considerable research studies were carried out to explore laser shock processing. High power laser induced damage in titanium nitride films was investigated by Mihac and Nenadoric. ${ }^{[1]}$ They showed that the damage threshold was a function of film thickness and had greater values for thicker films due to the enhanced optical absorption. A model for the analysis of residual stresses induced by laser coatings was presented by Freitas et al. ${ }^{[2]}$ They indicated that the predictions of the model agreed well with the experimental findings. The characterization and modeling of laser shock waves were investigated by Darguery. ${ }^{[3]}$ The fatigue behavior of the metallic substrates subjected to a laser shock processing was studied by Gerland et al. ${ }^{[4]}$ The physical processes involved in laser shock processing were studied by Pegre et al. ${ }^{[5]}$ They presented the new areas of laser shock processing, and the detrimental features of laser shock processing were discussed with the objective of defining an industrially suitable laser shock configuration.

The current study is carried out to explore the laser shock processing of steel. The numerical solutions for temperature and recoil pressure generation are presented. The elastic and plastic waves propagation in the substrate material was formulated and numerical solution for the momentum equation is obtained. An experiment was conducted to generate a laser shock hardened substrate. Scanning electron microscopy (SEM) and transmission electron microscopy (TEM) were carried out for surface morphology and metallurgical examination of laser shock processed regions. Microhardness tests across the laser-processed region were realized.

\section{Heat Transfer and Recoil Pressure}

The Fourier heating model is introduced when obtaining the temperature field during the laser heating pulse. In the analysis, evaporation of the surface is considered while liquid ejection and nucleate boiling in the liquid phase are neglected. The thermal properties of the substrate are considered to be constant, and the radiative cooling of the surface is ignored.

The heat transfer equation including the evaporation due to a laser heating pulse can be written as:

$$
\begin{aligned}
k \frac{\partial^{2} T(x, y)}{\partial x^{2}} & +\rho C_{\mathrm{p}} V \frac{\partial T}{\partial x}+I_{\mathrm{o}}\left(1-r_{\mathrm{f}}\right) \exp (-\beta t) \delta \exp (-\delta x) \\
& =\frac{\partial}{\partial t}\left[\rho C_{\mathrm{p}} T(x, t)\right]
\end{aligned}
$$

where $V$ is the recession velocity of the surface, which can be determined from the energy balance at the surface, ${ }^{[6]}$ i.e.,

$V=\frac{I_{\mathrm{o}}}{\rho\left[C_{\mathrm{p}} T+L_{\mathrm{ev}}\right]}$

An exponential time varying heating pulse is considered $\left[I_{\mathrm{o}}\left(1-r_{\mathrm{f}}\right) \exp (-\beta t]\right.$. The initial condition is:

At $t=0 ; \quad T(x, 0)=0$
The boundary conditions are:

At the surface; $\left.\frac{\partial T(x, t)}{\partial x}\right|_{x=0}=\frac{\rho}{k} V L_{\mathrm{ev}}$

and

At $x=\infty ; \quad T(\infty, t)=0$

where $L_{\mathrm{ev}}$ is the latent heat of evaporation.

The recoil pressure can be calculated after considering the rate of momentum change across the receding surface. ${ }^{[7]}$ The rate of momentum, which occurs across the receding surface, is

$A \cdot P_{\mathrm{r}}=\dot{m} V_{\mathrm{ev}}$

where $A$ is the area of irradiated surface, $P_{\mathrm{r}}$ is the recoil pressure, $\dot{m}$ is the rate of mass flow and $V_{\mathrm{ev}}$ is the evaporating front velocity, which can be formulated from the Maxwell's law for the velocity distribution of molecules, ${ }^{[6]}$ i.e.,

$V_{\mathrm{ev}}=\sqrt{\frac{k_{\mathrm{B}} T_{\mathrm{s}}}{2 \pi m}} \exp \left(-\frac{m L_{\mathrm{ev}}(T)}{k_{\mathrm{B}} T_{\mathrm{s}}}\right)$

where $m$ is the mass of an atom.

Equation 3 can be rearranged and the recoil pressure written as:

$P_{\mathrm{r}}=\frac{\dot{m}}{A} V_{\mathrm{ev}}$

When the irradiated energy is absorbed by the substrate material, some part of the absorbed energy is consumed as heat transferred to the solid substrate due to conduction and the other part is used for the evaporation of the surface. Consequently, energy dissipated for evaporation per unit time per unit area results in releasing molecules from the surface and acceleration of the vapor molecules to $V_{\text {ev }}$, i.e., the energy dissipated per unit time due to evaporation is the sum of latent heat of evaporation and the kinetic energy of the evaporated molecules per unit time. This yields:

$I_{\mathrm{ev}}=\frac{\dot{m}\left(\frac{1}{2} V_{\mathrm{ev}}^{2}+L_{\mathrm{ev}}\right)}{A}$

Substitution of $\frac{\dot{m}}{A}$ in the above equation yields:

$I_{\mathrm{ev}}=\frac{P_{\mathrm{r}}}{V_{\mathrm{ev}}}\left(\frac{1}{2} V_{\mathrm{ev}}^{2}+L_{\mathrm{ev}}\right)$

Therefore, the recoil pressure can be written as:

$P_{\mathrm{r}}=\frac{I_{\mathrm{ev}} V_{\mathrm{ev}}}{\left(\frac{1}{2} V_{\mathrm{ev}}^{2}+L_{\mathrm{ev}}\right)}$ 
The laser power intensity dissipated due to evaporation is

$$
I_{\mathrm{ev}} \simeq I_{\mathrm{o}}-\dot{q}_{\mathrm{solid}}
$$

where $\dot{q}_{\text {solid }}$ is the energy dissipated in the solid phase. It should be noted that the energy dissipated due to melting is considerably smaller than the energy dissipated during evaporation.

The vapor pressure predicted from Eq 5 is validated using the equation given in the previous study, ${ }^{[8]}$ i.e.,

$P_{\mathrm{s}}=1.82 \times 10^{-3} \frac{\sqrt{C_{\mathrm{p}} T_{\mathrm{ev}}} I_{0}}{\left[C_{\mathrm{p}}\left(T_{\mathrm{ev}}-T_{\mathrm{o}}\right)+L_{\mathrm{v}}\right]}$

Equation 6 is expressed in units of Bar. When the results of $\mathrm{Eq}$ 5 and 6 were compared, it they were in good agreement.

\subsection{Method of Solution}

To discretize the governing equations, a finite difference approach is introduced. The details of the numerical scheme are given in Ref 9. To compute the temperature field and relative positions of solid-vapor interface, an explicit scheme (a time marching scheme) is used; i.e., using the initial conditions, the temperature in the whole domain is calculated for the following time steps with their respective conditions. This process is repeated for the next time step and $V \neq 0$ is introduced wherever the temperature reaches the boiling temperature of the substrate material. The stability criteria due to the time increment are considered for a stable solution; therefore, the time increment is limited by:

$$
\left(\frac{2 k}{\rho c_{\mathrm{p}} \Delta z^{2}}\right) \Delta t \leq 1
$$

Time increment satisfying the stability criteria for the finest grid is calculated as $10^{-13} \mathrm{~s}$. A computer program based on explicit scheme is developed to compute the temperature field. The material properties used in the simulations are given in Table 1.

\section{Wave Analysis}

When the free surface of the solid substrate is subjected to an impact force due to a suddenly generated pressure, an elastic-plastic wave, which propagates into the solid, is generated. The wave amplitude is composed of two parts, which are the amplitudes greater and less than the yield stress. The amplitude of the wave greater than yield stress results in the plastic deformation inside the substrate. As soon as the impact load is released from the surface, a loading wave is initiated that propagates through the substrate. The point where the unloading wave intersects the elastic-plastic wave determines the depth of the plastic region formed in the substrate. The mathematical analysis governing the wave propagation is given in Ref 10; therefore, only the basic equations are given below. The local momentum balance for homogeneous mass of a solid can be written as ${ }^{[10]}$ :

$\rho \frac{\partial u}{\partial t}=\frac{\partial \sigma}{\partial x}$

where $u$ is the velocity in the $x$-direction, $\sigma$ is the normal stress, $\sigma$ is the density of the substrate, and $t$ is the time. When the displacement is denoted by $U$ and the strain by $\varepsilon$, the following relations can be written:

$u=\frac{\partial U}{\partial t}$ and $\varepsilon=\frac{\partial U}{\partial x}$

The integrability condition of the substrate results in

$\frac{\partial \varepsilon}{\partial t}=\frac{\partial u}{\partial x}$

For a linear elastic material, Hooke's law gives

$\sigma=E \varepsilon$

where $E$ is Young's modulus. Substitution of $\sigma$ into Eq 7 yields

$\frac{\partial w}{\partial t}=A \frac{\partial w}{\partial x}$

where

$w=\left(\begin{array}{c}\rho u \\ \varepsilon\end{array}\right)$ and $A+\left(\begin{array}{cc}0 & E \\ \frac{1}{\rho} & 0\end{array}\right)$

Therefore:

$\frac{\partial w}{\partial t}=\frac{\partial f}{\partial x}$

where $f=(\sigma, u)^{T}$

In the case of elastic-plastic material, the loading history of the boundary and work hardening of the substrate should be considered. To account for the elastic-plastic condition, the stress-strain relation can be written as ${ }^{[10]}$ :

Table 1 Properties of Stainless Steel Used in the Present Study

\begin{tabular}{lccccccc}
\hline $\begin{array}{l}\boldsymbol{\delta}, \\
\mathbf{l} / \mathbf{m}\end{array}$ & $\begin{array}{c}\boldsymbol{\alpha}, \\
\mathbf{m} / \mathbf{s}\end{array}$ & $\begin{array}{c}\boldsymbol{C}_{\mathbf{p}}, \\
\mathbf{J} / \mathbf{k g K}\end{array}$ & $\begin{array}{c}\boldsymbol{\gamma}, \\
\mathbf{m}\end{array}$ & $\begin{array}{c}\boldsymbol{k}, \\
\mathbf{W} / \mathbf{m K}\end{array}$ & $\begin{array}{c}\boldsymbol{L}_{\mathbf{m}}, \\
\mathbf{k J} / \mathbf{m o l}\end{array}$ & $\begin{array}{c}\boldsymbol{L}_{\text {ev }}, \\
\mathbf{k J} / \mathbf{m o l}\end{array}$ & $\begin{array}{c}\boldsymbol{T}_{\mathbf{m}}, \\
\mathbf{K}\end{array}$ \\
\hline $6.16 \times 10^{7}$ & $0.227 \times 10^{-4}$ & 460 & $10^{-9}$ & 80.3 & 13.8 & 349.5 & 1892 \\
\hline
\end{tabular}


$d \varepsilon=\frac{1+h}{E} d \sigma$

where $h=h(\kappa)$ is defined as the plastic factor, which has the following conditions:

$h=0$ at $|\sigma+d \sigma| \leq \kappa$

and $h=\frac{E}{E_{\mathrm{p}}(\kappa)}-1$ at $|\sigma+d \sigma|>\kappa$

Substitution of $d \varepsilon$ into $\mathrm{Eq} 11$ yields:

$A \frac{\partial f}{\partial t}=\frac{\partial f}{\partial x}$

where:

$A=\left(\begin{array}{cc}0 & \rho \\ \frac{1+h}{E} & 0\end{array}\right)$ and $\rho=\left(\begin{array}{l}\sigma \\ u\end{array}\right)$

\subsection{Method of Solution}

The elastic-plastic boundaries in the time and space $(x, t)$ domain are established by obtaining the first plastic $\left(\kappa / \kappa_{\mathrm{o}}\right)$ wave and the elastic unloading wave. A semi-infinite body, which obeys a linearly elastic, power-law work-hardening plastic stress-strain relationship is considered, which may be written as:

$\frac{1}{E(\kappa)}=\frac{\alpha}{E}\left(\frac{\kappa}{\kappa_{\mathrm{o}}}\right)^{\alpha-1}$

The solid body is assumed to be at rest at $t<0$, which in turn yields the initial conditions for $u$ and $\sigma$ that are zero. The boundary condition at $x=0$ includes a rectangular impact load, i.e.,

$\sigma(0, t)=P_{\mathrm{r}}(1 \mathrm{GPa})$ for $0 \leq t \leq t_{\mathrm{p}} \mathrm{ns}$

$\sigma(0, t)=P_{\mathrm{r}}(0 \mathrm{GPa})$ for $t_{\mathrm{p}} \mathrm{ns} \leq t \leq \infty$

where $t_{\mathrm{p}}$ is twice the pulse length $\left(t_{\mathrm{p}}=2 \times 9 \mathrm{~ns}\right)$. It should be noted that due to the overlay placed at the workpiece surface in the experiment, $t_{\mathrm{p}}$ is set to twice the pulse length as suggested in the previous study. ${ }^{[8]}$ A computer program was developed to solve the governing stress wave equation numerically using the Godunov scheme. ${ }^{[11]}$ The numerical procedure consists of three steps: (1) Solving the Riemann problem to obtain the solution for the fan-shaped simple wave regions on both sides of the interface, (2) Calculating $\bar{f}_{j+1 / 2}$, and (3) updating $w_{j}^{n+1}$ for $u$ and $\sigma$. The mechanical properties used in the simulations are given in Table 2.
Table 2 Mechanical Properties of Steel Used in the Present Study

\begin{tabular}{lccccc}
\hline $\begin{array}{l}\boldsymbol{\sigma}_{\mathbf{y}} \\
\mathbf{M P a}\end{array}$ & $\begin{array}{c}\boldsymbol{E} \\
\mathbf{k P a}\end{array}$ & $\begin{array}{c}\boldsymbol{\rho g} \\
\mathbf{k N} / \mathbf{m}^{\mathbf{3}}\end{array}$ & $\boldsymbol{\alpha}$ & $\begin{array}{c}\boldsymbol{T}_{\mathbf{t}} \\
(\mathbf{n s})\end{array}$ & $\boldsymbol{v}$ \\
\hline 230 & 207 & 76.5 & 2 & 40 & 0.33 \\
\hline
\end{tabular}

Table 3 Elemental Composition of Stainless Steel

\begin{tabular}{lc}
\hline Contents & Stainless Steel \\
\hline $\mathrm{Fe}$ & Balance \\
$\mathrm{C}(\max )$ & 0.08 \\
$\mathrm{Mn}$ & 2 \\
$\mathrm{P}$ & 0.04 \\
$\mathrm{~S}$ & 0.03 \\
$\mathrm{Si}$ & 0.75 \\
$\mathrm{Ni}$ & 10 \\
$\mathrm{Mo}$ & 2 \\
\hline
\end{tabular}

\section{Experimental}

To irradiate the workpiece surfaces a Nd:YAG laser was used with an excitation source of $355 \mathrm{~nm}$ high power laser beam, which is generated from the third harmonic of Nd:YAG laser, model GCR 250 (Spectra Physics, Mountain View, CA). The third harmonic radiation provides improved absorption of the incident power by the substrate material, since reflectivity of the surface reduces considerably as the wavelength reduces. The laser delivers nominal output energy of $450 \mathrm{~mJ}$ within a pulse length of about $8 \mathrm{~ns}$. The repetition rate of the laser pulses is $10 \mathrm{~Hz}$. To increase the laser output pulse intensity, a focusing lens with a nominal focal length of $100 \mathrm{~mm}$ was used. The diameter of the heated spot was kept at about $2.5 \mathrm{~mm}$ at the workpiece surface. Fifteen consecutive pulses were fired during the shock processing of the surface. An overlay of $0.1 \mathrm{~mm}$ thickness was mounted on the workpiece surface to enhance the recoil pressure developed at the interface. It should be noted that using an overlay increases substantially the magnitude of the recoil pressure. Moreover, during the ablation, a local melting can produce high residual stress levels in the surface vicinity of the workpieces; i.e., the confined regimen develops.

A commercial 316 stainless steel (chemical composition is given in Table 3) was cut into a rectangular shape: $12 \times 30 \times$ $2 \mathrm{~mm}^{3}$ (width $\times$ length $\times$ thickness). The workpieces were cleaned ultrasonically before the experiments. The crosssections of the workpieces were obtained and etched after the laser treatment process.

SEM (JEOL JSM-T300, Tokyo, Japan) was used for microstructural analysis while microhardness across the workpiece surfaces was measured with Vickers microhardness tester (Puhlar Micromet 3). During the microhardness tests a load of $300 \mathrm{~g}$ was used. TEM (JEOL 2527-TEM SCAN, Tokyo, Japan) was used to obtain the TEM micrographs for determining the dislocation densities in the laser-shocked zone.

The laser workpiece interaction was recorded continuously using a charge-coupled device (CCD) camera (Sony CCD TRV51E, Japan). The camera was located to monitor the lon- 
gitudinal section of the workpiece. This arrangement allowed a close view of the initiation of the surface evaporation, and plasma formation and expansion.

\section{Results and Discussion}

Laser ablation of stainless steel and laser shock hardening of the surface were investigated. The recoil pressure generated at the surface during the ablation was determined numerically. The loading force generated due to recoil pressure was considered as a force generating a plastic zone in the surface region of the substrate material.

Figure 1 shows the recoil pressure generated at the surface. The recoil pressure increases considerably with increasing power intensity; in which case, high temperature at the surface occurs with increasing power intensity. This, in turn, acceler-

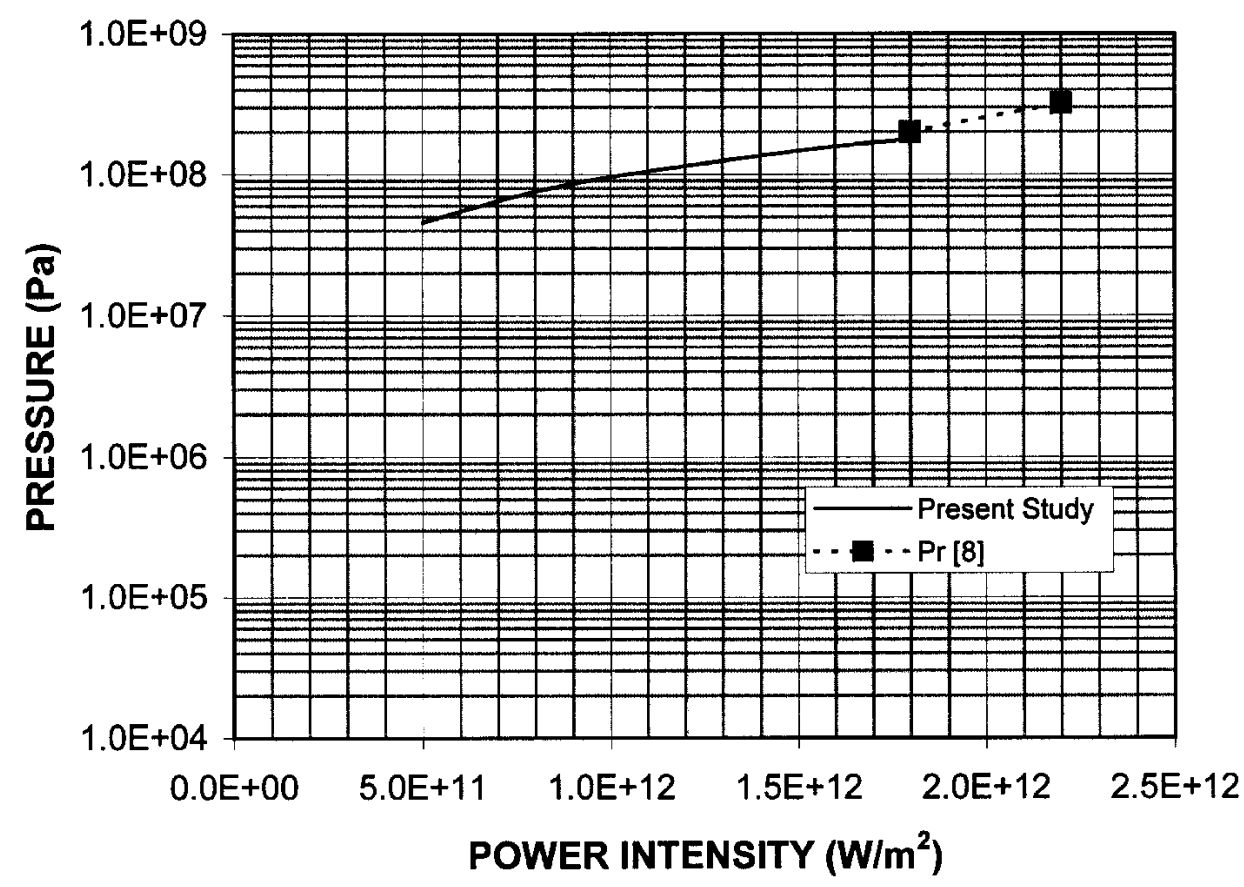

Fig. 1 Recoil pressures with power intensity

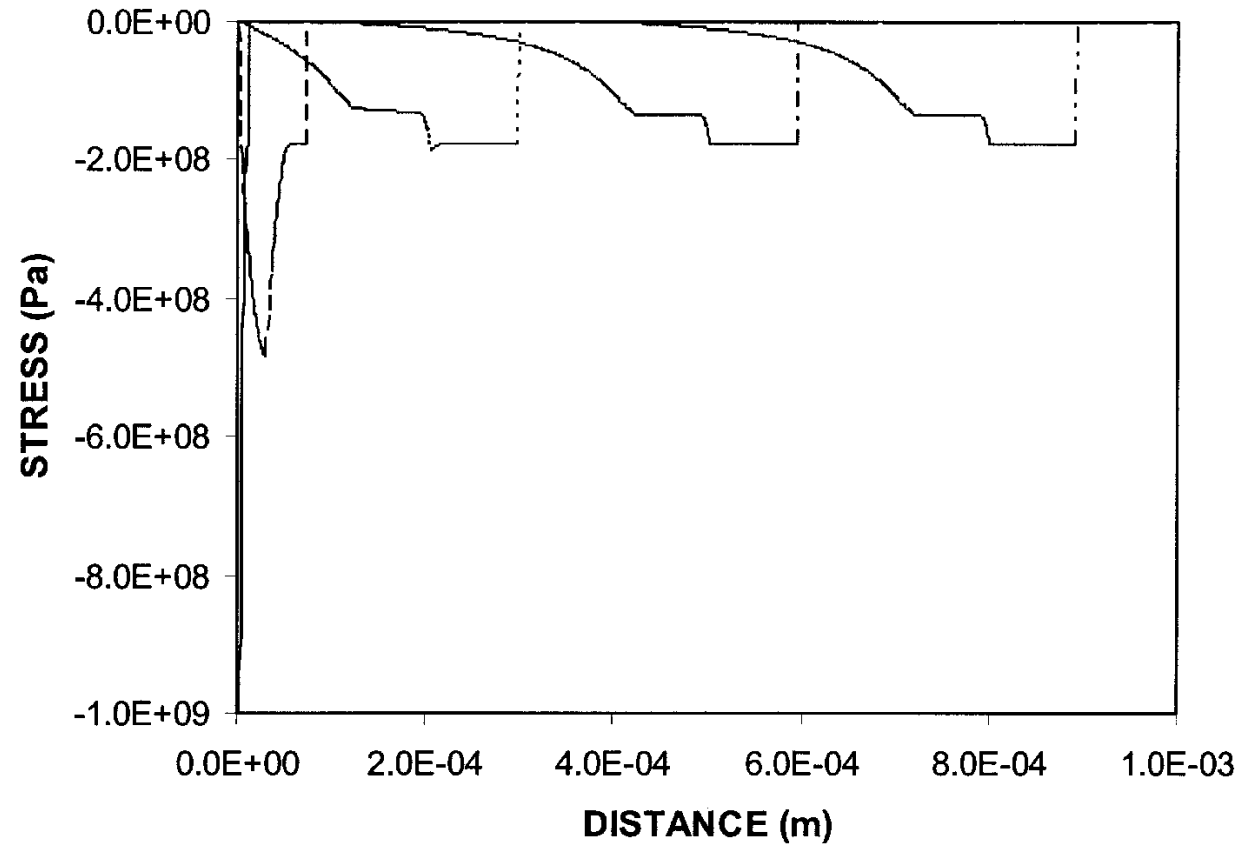

Fig. 2 Stress wave propagation for stainless steel with pressure $=0.8 \mathrm{GPa}$, pulse length $=8.0 \mathrm{~ns}$, and $d t=0.05 \mathrm{~ns}$ 

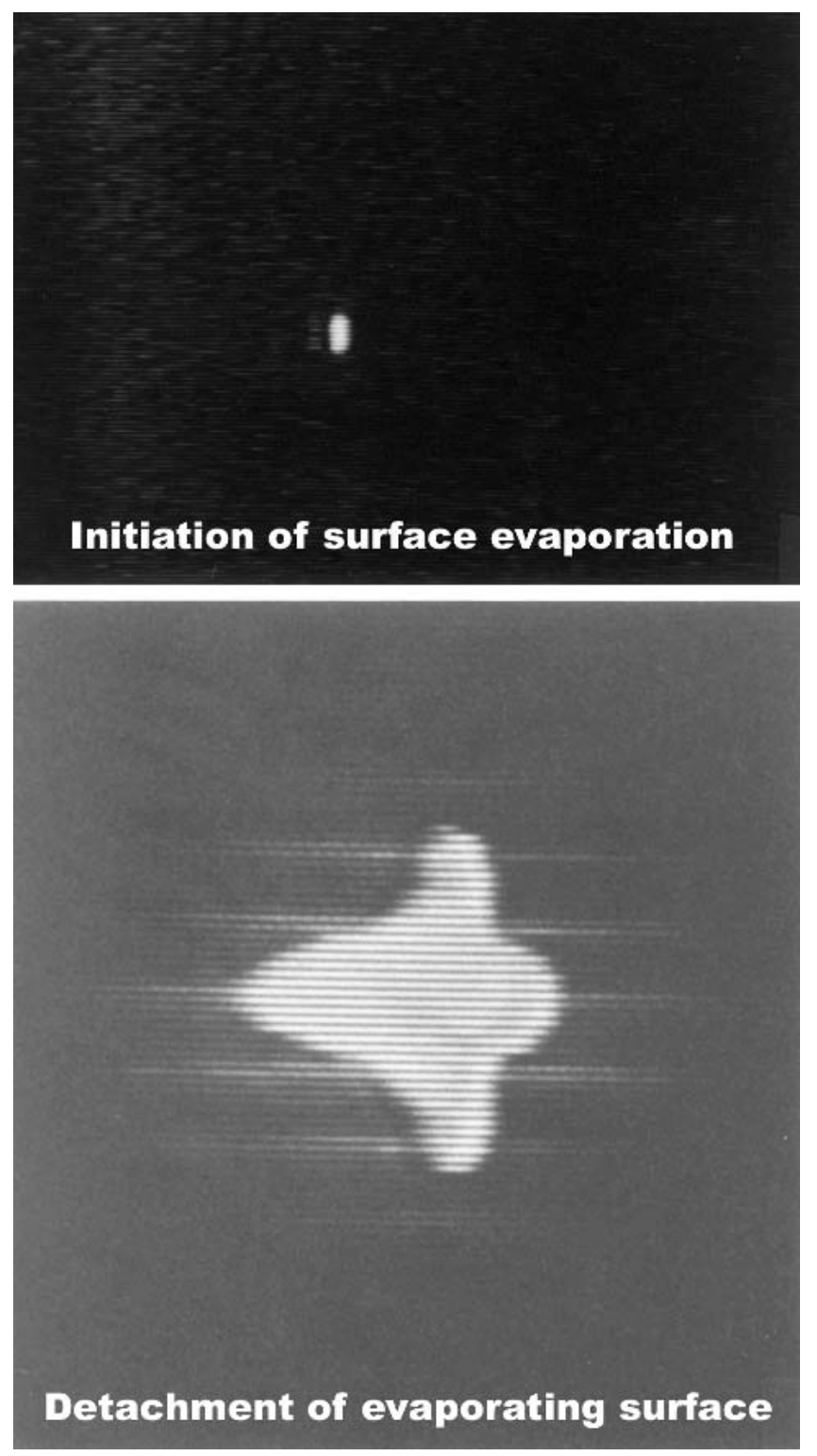

Fig. 3 Photographs of evaporating surface

ates the vapor front as well as recession velocities of the surface. Consequently, a considerably high magnitude of recoil pressure is generated due to the momentum change in the direction of flow. When comparing the experimental values with the present predictions, both results are in agreement.

Figure 2 shows the stresses developed inside substrate material due to propagation of the plastic wave generated by the recoil pressure at the workpiece surface. The magnitude of stress attains high values in the workpiece surface vicinity in the early heating period. The stress due to plastic wave propagation is compressive in nature. Moreover, once the evaporation ceases, recoil pressure reduces to zero and the unloading wave with a higher speed $\left(c_{\mathrm{o}}\right)$ than the plastic wave propagates towards the substrate material. The unloading wave catches the plastic wave and reduces the plastic wave amplitude significantly. Consequently, the plastic wave terminates at the point

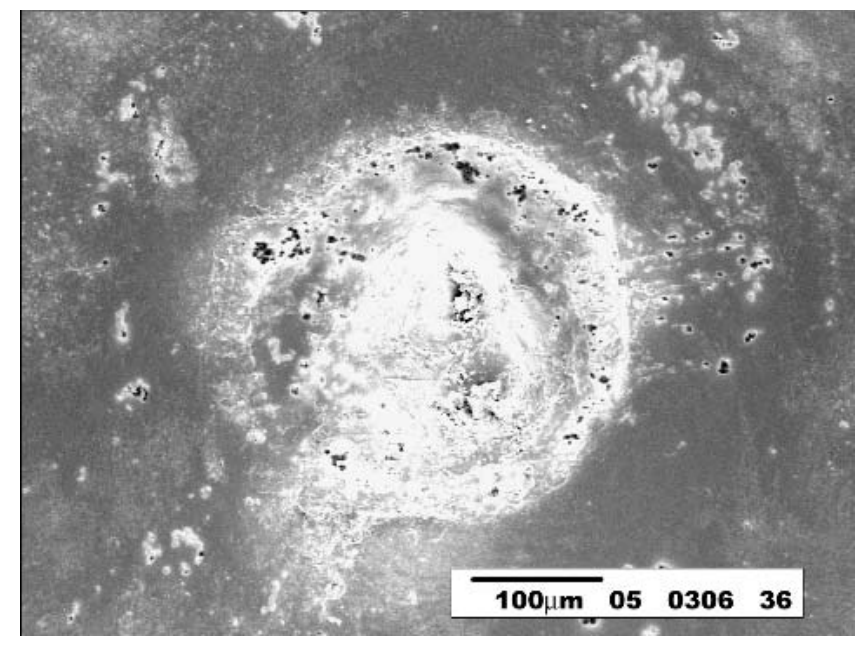

Fig. 4 SEM micrograph of laser shock processed surface

where the plastic and the unloading elastic waves meet. The time where these two waves interact determines the time taken for plastic region formation. In the present case, it is of the order of $1.2 \times 10^{-7} \mathrm{~s}$, and the corresponding depth of plastic region is in the order of $400 \mu \mathrm{m}$.

The photographs of initiation and progression of evaporating front are shown in Fig. 3. The evaporating front expands rapidly, detaches from the surface and generates a shock front propagating into the stir air. The detachment of the evaporating front indicates that the considerable pressure generation across the vapor-solid interface and the evaporating front partially blocks the incident beam reaching the substrate surface. This reduces the material removal from the surface. Moreover, the evaporated front expands rapidly due to the absorbed incident beam, which increases the pressure differential between the evaporating front and its surroundings. This allows more laser power to reach the surface and restarts the ablating process. This is consistent with previous work. ${ }^{[12]}$

Figure 4 shows the top view of the laser treated workpiece surface. The size of the surface region ablated is on the order of $2.5 \mathrm{~mm}$. A shallow cavity is formed at the surface. The laser evaporated and shock regions can be identified from the view of the surface. The formation of waviness and small recession of the surface are due to evaporation of the surface and the plastic deformation in the surface vicinity. Moreover, nonexistence of the liquid layer at the surface also indicates that considerable portion of the incident laser energy is transferred to surface ablation process. The excessive compressive stress and thermal effect due to the confined regimen generate a plastic zone in the surface region, as indicated in the previous study. ${ }^{[8]}$

Figure 5 shows the microhardness distribution across the heated surface. Microhardness is almost 1.8 times that of the base substrate hardness in the central region of irradiated spot. The decay of the hardness across the heated spot towards its edge is due to the power intensity distribution at the surface; i.e., power intensity reduces from heated spot center to its edge. The increase in hardness is associated with the dislocation generated in the surface region of the irradiated spot during the heating process. This can also be seen from TEM micrograph 
in Fig. 6. The dark areas on the micrograph are representative of dislocations (dislocation strain contrast). From these images, the dislocation density can be determined and is on the order of $2-8 \times 10^{11} \mathrm{~cm}^{-2}$, which in turn results in a hardness increase of the treated region of 1.8 times that of the base metal. In this case, the relation between the hardness and dislocation density, which was developed previously, ${ }^{[13]}$ is used, since the dislocation density is known. The equation for the hardness due to dislocation is

$$
H=145+2.1 \times 10^{-4} \sqrt{P}
$$

where 145 is the hardness of the base material, $2.1 \times 10^{-4}$ is constant for steel, and $P$ is the dislocation density. Therefore, substituting the dislocation density in the above equation, the hardness values in the ablated region is on the order of $250 \mathrm{HV}$, which is in good agreement with the measurements (Fig. 5).

\section{Conclusions}

Laser shock hardening of steel surface was considered. The recoil pressure generated at the liquid-vapor interface was formulated and elasto-plastic wave analysis due to the recoil pressure loading at the surface was carried out. An experiment was conducted to ablate the workpiece surfaces. SEM was used to examine the morphological changes after the ablation process, while TEM was performed to determine the dislocation density in the plastic region of the substrate material. Microhardness

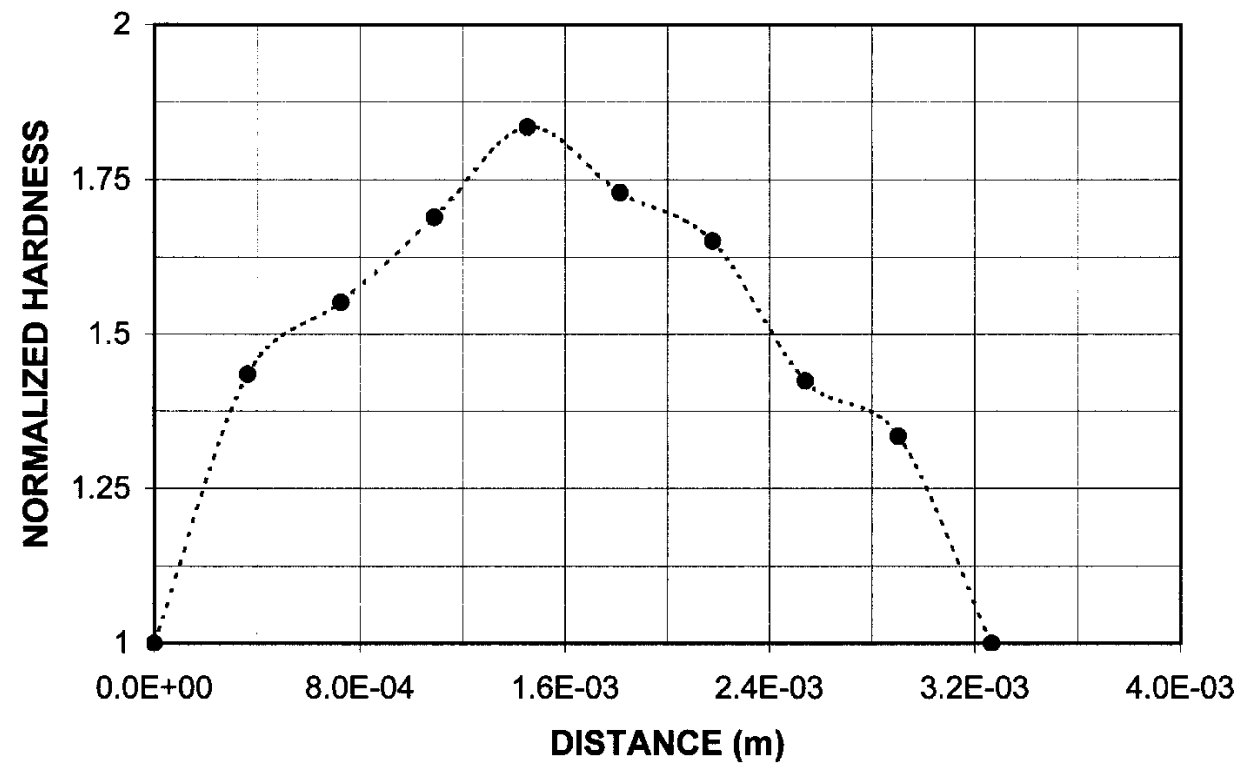

Fig. 5 Normalized microhardness distribution across the irradiated spot
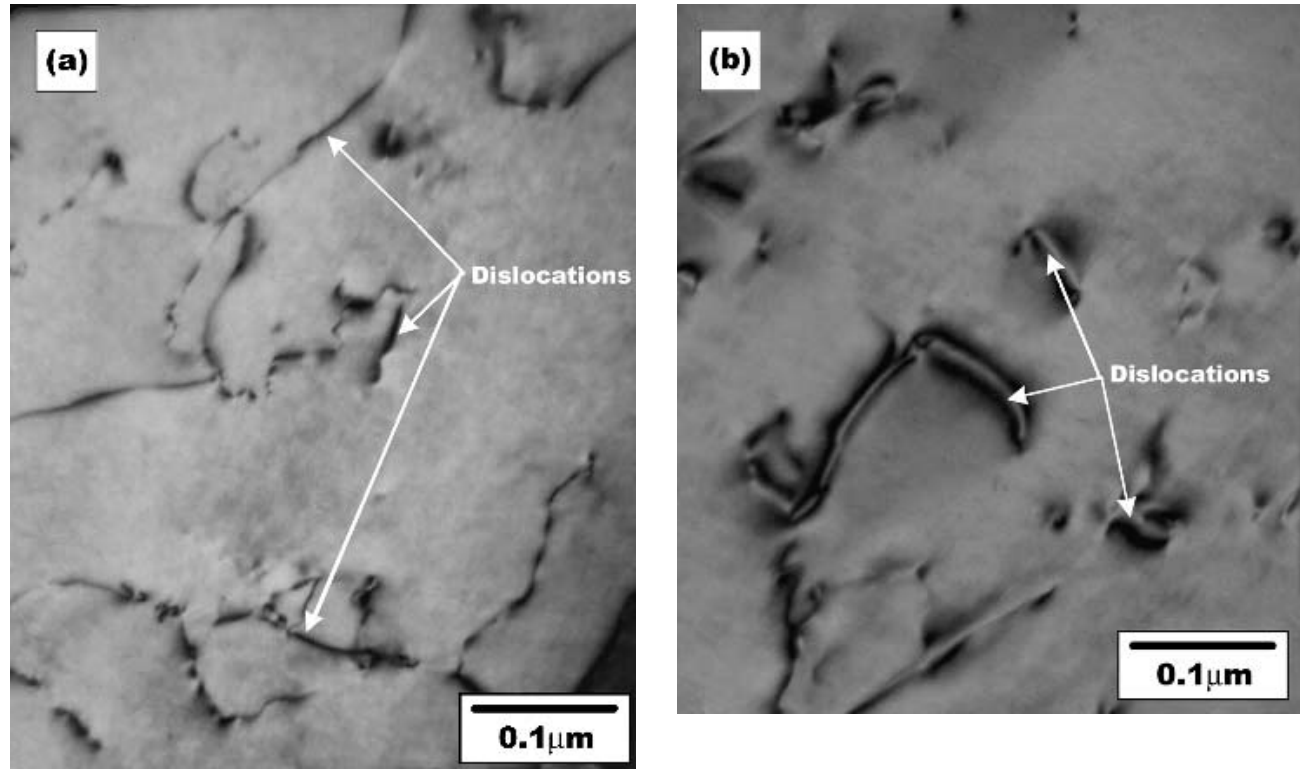

Fig. 6 TEM micrograph of laser shock processed region, magnification: 150 000x 
measurements were made across the ablated surfaces. In general, microhardness values as high as 1.8 times that of the base hardness of the substrate material were achieved at the ablated surface. The hardening of the workpiece in the plastic region occurred due to the dislocation generated due to the propagation of the high amplitude plastic wave. SEM micrographs showed that evaporation is the sole mechanism governing the mass removal from the surface during the ablation process. In this case, recoil pressure in the order of $0.8 \mathrm{GPa}$ was generated, which in turn initiated the generation and propagation of the plastic waves into the substrate material. Once the recoil pressure diminished due to cessation of the evaporation process, the unloading wave with its higher speed than the plastic wave initiated and propagated into the workpiece. At the time these waves interacted, the plastic wave diminished. Consequently, the depth of plastic zone was limited to the depth where the two waves interact. In the present case, the time at which these two waves interact was on the order of $1.2 \times 10^{-7} \mathrm{~s}$, and the depth where they interact was on the order of $400 \mu \mathrm{m}$.

\section{Acknowledgment}

The authors acknowledge the support of King Fahd University of Petroleum and Minerals, Dhahran, Saudi Arabia for this work.

\section{References}

1. T. Mihac and T. Nenadovic: "Laser Induced Damage in Thin Ti and TiN Films," Vacuum, 1990, 40, pp. 139-41.

2. M. Freitas, M.S. Pereira, M. Michaud, and D. Pantelis: "Analysis of
Residual Stresses Induced by Laser Processing," Mater. Sci. Eng., 1993, A167, pp. 115-22.

3. P. Darquey: "Ondes de choc et acceleration de feuilles minces par impulsion laser en interaction confinee. Test d'application a la compaction dynamique de poudres metalliguies," Ph.D. Thesis, University of Poitiers, France, 1989 (In French).

4. M. Gerland, M. Hallouin, and H.N. Presles: "Comparison of Two New Surface Treatment Processes, Laser-Induced Shock Waves and Primary Explosive: Application to Fatigue Behavior," Mater. Sc. Eng., 1992, Vol. A156, pp. 175-82.

5. P. Peyre and R. Fabbro: "Laser shock Processing: A Review of the Physics and Applications," Opt. Quantum Electron., 27, 1995, pp. 1213-29,

6. B.S. Yilbas, A. Sahin, and R. Davies: "Laser Heating Mechanism Including Evaporation Process Initiating the Laser Drilling," Int. J. Mach. Tools Manufact., 1995, 35(7), pp. 1047-62.

7. B.S. Yilbas and M. Sami: "Liquid Ejection and Possible Nucleate Boiling Mechanisms in Relation to Laser Drilling Process," J. Phys.: Part D, J. Appl. Phys., 1997, 30, pp. 1996-2005.

8. P. Peyre, R. Fabbro, L. Berthe, and C. Dubouchet: "Laser Shock Processing of Materials, Physical Processes Involved and Examples of Applications," J. Laser Appl, 1996, 8, pp. 135-41.

9. G.D. Smith: Numerical Solution of Partial Differential Equations: Finite Difference Methods, 3rd ed, Clarendon Press, Oxford, United Kingdom, 1985.

10. L. Xiao: Numerical Computation of Stress Waves in Solids, Akademie Verlag GmbH, Berlin, Germany, 1996.

11. S.K. Gudonov: "A Finite Difference Method for the Numerical Computation of Discontinuous Solutions of Equations of Fluid Dynamics," Mat. Sb., 1959, 1, pp. 271-306.

12. B.S. Yilbas, M. Sami, and A. Al-Ferayedhi: "Closed Form and Numerical Solutions to the Laser Heating Process," Proc. Istn. Mech. Engrs., Part C; J. Mech. Eng. Sci., 1998, 212, pp. 141-51.

13. J.P. Chu, J.M. Rigsbee, G. Banas, H.E. Elsayed-Ali: "Laser-Shock Processing Effects on Surface Microstructure and Mechanical Properties of Low Carbon Steel," Mater. Sci. Eng., 1999, 260A, pp. 260-68. 